\title{
Wrist Fracture
}

National Cancer Institute

\section{Source}

National Cancer Institute. Wrist Fracture. NCI Thesaurus. Code C80517.

Traumatic injury to the wrist joint in which the continuity of a wrist bone is broken.

Symptoms include wrist pain, swelling, and deformity of wrist. 